\title{
High-frequency forcing of a turbulent axisymmetric wake
}

\author{
Anthony R. Oxlade ${ }^{1}$, Jonathan F. Morrison ${ }^{1} \dagger$, \\ Ala Qubain ${ }^{2}$ and Georgios Rigas ${ }^{1}$ \\ ${ }^{1}$ Department of Aeronautics, Imperial College, London, SW7 2AZ, UK \\ ${ }^{2}$ Mustakbal Clean Tech. P.O Box 5272 Amman, Jordan 11183
}

(Received ?; revised ?; accepted ?. - To be entered by editorial office)

A high-frequency periodic jet, issuing immediately below the point of separation, is used to force the turbulent wake of a bluff axisymmetric body, its axis aligned with the free stream. It is shown that the base pressure may be varied more-or-less at will: at forcing frequencies several times that of the shear-layer frequency, the time-averaged areaweighted base pressure increases by as much as $35 \%$. An investigation of the effects of forcing is made using random and phase-locked two-component PIV, and modal decomposition of pressure fluctuations on the base of the model. The forcing does not target specific local or global wake instabilities: rather, the high-frequency jet creates a row of closely spaced vortex rings, immediately adjacent to which are regions of large shear on each side. These shear layers are associated with large dissipation and inhibit the entrainment of fluid. The resulting pressure recovery is proportional to the strength of the vortices and is accompanied by a broadband suppression of base pressure fluctuations associated with all modes. The optimum forcing frequency, at which amplification of the shear layer mode approaches unity gain, is roughly five times the shear-layer frequency.

\section{Key words:}

\section{Introduction}

Turbulent entrainment (Townsend 1976) is a fundamental process governed by both local conditions of the moving turbulent/non-turbulent interface - the viscous "superlayer" - as well as other non-local factors. The superlayer is characterised by an approximate balance of turbulent advection of energy and its viscous diffusion, the equivalent physical processes having been described, respectively, as "engulfment" (Turner 1986) and "nibbling" (Corrsin \& Kistler 1955). Modern experimental techniques have enabled study of the relative importance of these fundamental processes involved in vorticity transport (Westerweel et al. 2005; Philip \& Marusic 2012): they are key in controlling the growth rates of free shear layers and therefore the reactive forces on the solid bodies producing them. Control of entrainment can therefore make many fluid-based systems more efficient: for example, its reduction in the wake of a bluff body will reduce the pressure (or "form") drag of that body. Here, we report a phenomenon in which a high-frequency jet is used to increase the base pressure of a bluff body from which separation occurs at a sharp trailing edge. Given that the drag of such a body is dominated by pressure drag, there is an associated reduction in the wake momentum flux deficit.

$\dagger$ Email address for correspondence: j.morrison@imperial.ac.uk 
A recent review of the subject by Choi et al. (2008) includes techniques that are either passive (for example, Heenan \& Morrison 1996, 1998; Strykowski \& Sreenivasan 1990; Parezanovic \& Cadot 2012) or active (Zaman \& Hussain 1981; Gaster et al. 1985; Kim \& Choi 2005; Vukasinovic et al. 2010), the latter with or without feedback (Kang \& Choi 2002; Dahan et al. 2012). Effective direct-wake control (as opposed to separation delay), whether in open or closed loop, requires an understanding of the large-scale organised structures and their interaction. These are geometry-specific: in the present study, we apply pulsed-jet blowing to the near-wake of an axisymmetric body, its axis aligned with the free-stream direction, and where turbulent separation occurs at a sharp trailing edge, immediately beneath which there is a small annular slit through which the jet emerges parallel to the free stream: see figure 1.

We initially reported a significant pressure recovery (Qubain 2009; Morrison \& Qubain 2009; Qubain \& Morrison 2009) over a narrow range of frequencies (at about five times the shear layer frequency) without a detailed understanding of how it occurs. The effect is generic in that it has been demonstrated for turbulent flow over a backward-facing step (Qubain 2007) as well as a bluff body of rectangular cross section (Cabitza 2013; Barros et al. 2014). Although the jet is of zero net-mass flux (Glezer \& Amitay 2002), we avoid the term "synthetic" jet because, as we show below, a time-averaged jet is not produced by the self-induced velocity of a vortex ring pair. We further observe that, crucially, the nonlinear coupling between the jet and the wake requires there to be a finite, jet-induced momentum flux which manifests itself as a change in pressure drag on the body. Here we show that a global instability can be controlled indirectly from a location where the separating boundary layer is convectively unstable, or where an "amplifier" is receptive to "extrinsic" disturbances (Huerre 2000).

A full description of the unforced wake in the present experiment is provided by Rigas et al. (2014b) who show that the coherent structures of the turbulent wake retain the symmetry-breaking properties of the laminar wake. Similarly, oscillatory bifurcations appearing at low Reynolds numbers (the ones at higher Reynolds numbers breaking reflectional symmetry) also appear in the turbulent wake as axisymmetric "bubble pumping" (Berger et al. 1990) and as "vortex shedding" (Achenbach 1974; Taneda 1978; Fuchs et al. 1979; Berger et al. 1990), a large-scale anti-symmetric oscillation. This has often been referred to as a "helical" mode when, in fact, as shown by Rigas et al. (2014b), periodic shedding is perturbed by a random variation of the azimuthal shedding angle on a very long timescale. They further show that, in a statistical sense, the broken symmetries are restored at much higher Reynolds numbers (Rigas et al. 2014a).

Monkewitz (1988) conducted a linear stability analysis for a family of axisymmetric wake (parallel flow) profiles: he showed that this asymmetric mode is absolutely unstable, and in agreement with experimental observation by Taneda (1978), he noted that it is most clearly observed somewhat downstream of the body. Grandemange et al. (2013) show that these characteristics are consistent with those of a wake from a threedimensional body of rectilinear geometry. Ho \& Huerre (1984) have identified the boundarylayer momentum thickness, $\theta$, as the appropriate length scale. A linear stability analysis of an initially laminar, plane mixing layer shows that maximum amplification occurs at a Strouhal number, $S t_{\theta}=f \theta / U_{\infty}=0.016$. For a turbulent separation, maximum amplification occurs at $S t_{\theta}=0.022-0.024$ (Zaman \& Hussain 1981; Ho \& Huerre 1984).

\section{Experimental Details}

Figure 1 shows a schematic of the model which is mounted in a closed-circuit wind tunnel, the working section of which measures $1.37 \mathrm{~m}$ x $1.22 \mathrm{~m} \times 3.0 \mathrm{~m}$. The contraction 

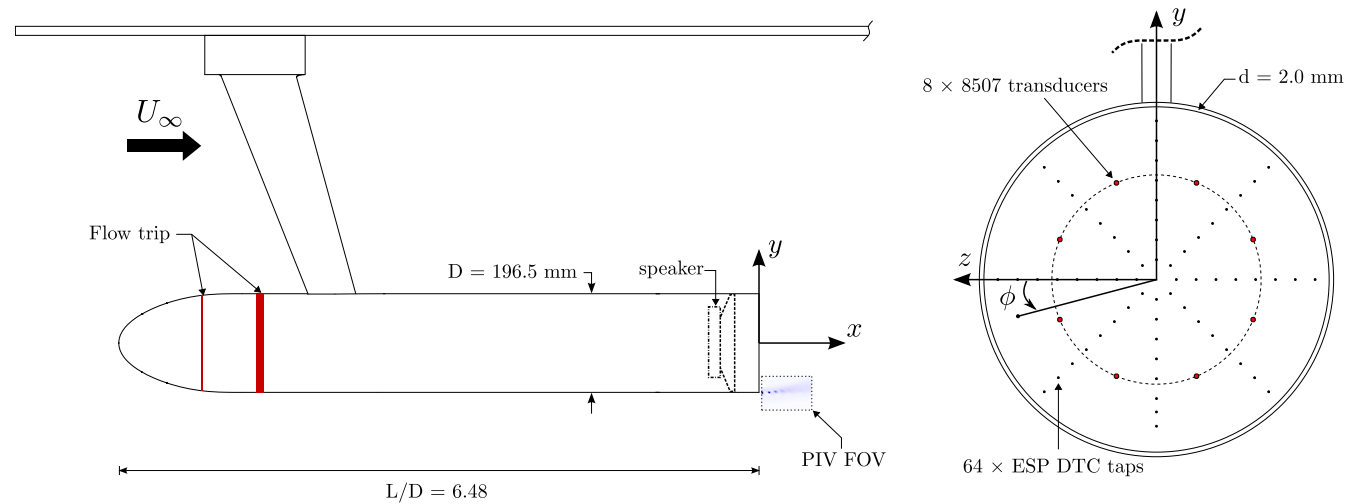

Figure 1. Schematic of the experimental model and PIV fields of view.

ratio is 4.92:1 and the free-stream turbulence intensity is less than $0.1 \%$. A PID controller with a set-point variation of less than $0.2 \%$ is used to maintain the free-stream velocity, $U_{\infty}=15 \mathrm{~ms}^{-1}$ (Reynolds number based on diameter of $R e_{D}=1.88 \times 10^{5}$, and at separation, $R e_{\theta}=2050$ ). The leading edge is a modified super ellipse with an aspect ratio of 2.5. The boundary layer is conditioned in two stages: the first is a $2 \mathrm{~mm}$ wide strip of 120 grit emery paper located at approximately the point of minimum surface pressure; the second is a $25 \mathrm{~mm}$ wide strip of 120 grit emery paper which serves to thicken the boundary layer. The boundary layer at separation was checked by a full traverse of about 50 data points: it shows an approximate log law region from which a wall friction velocity, $u_{\tau}=0.68 \mathrm{~ms}^{-1}$ is estimated. The shape factor, $H=1.34$.

A high-fidelity speaker mounted inside the model is used to generate a pulsed jet of variable frequency and amplitude. The jet issues in the free-stream direction from a $2.0 \mathrm{~mm}$ wide annular slit, located $1.0 \mathrm{~mm}$ below the trailing edge. The choice of slit width was based on preliminary measurements and chosen to match the boundary layer momentum thickness, $\theta=2.14 \mathrm{~mm}$. In order that jet disturbances are amplified as much as possible, the slit was placed as close as possible to the point of separation. A BEYMA 6MI100 mid-range driver was chosen for its high sensitivity (97 dB) and nominal power rating $(250 \mathrm{~W})$ to maximise the parameter space over which the pressure recovery can be studied. A harmonic forcing signal is generated by the control code and converted to an analogue signal by the 16-Bit D/A converter sampling at $800 \mathrm{kHz}$. A QSC RMX 850 high fidelity power amplifier drives the loudspeaker. The jet velocity was calibrated with a single hot wire against speaker-chamber pressure, the amplitude of which was also maintained constant using a PID controller, with a set-point variation for the speakerchamber pressure of less than $0.15 \%$. The jet centre-line velocity, $u_{f}$, is quantified in quiescent flow using a hot wire placed at the orifice: figure 2 shows the unrectified signal which is de-rectified by a simple peak/trough detection algorithm. $u_{j}$ is defined as the amplitude of the Fourier component of the jet centre-line velocity at the forcing frequency. This measure is preferable to one based solely upon the peak jet velocity (as used by Qubain 2009): firstly, it distinguishes between the flux at the forcing frequency and other frequency components which occur at larger forcing amplitudes, see figure 2; secondly, the hot wire rectifies the velocity with an unknown sensitivity to negative velocities. Since it is difficult to distinguish the efflux (blowing) from influx (suction), use of a Fourier amplitude reduces the uncertainty associated with the mis-identification of suction peaks. At forcing amplitudes used in this experiment, the jet turbulence intensity (as a deviation from a pure sinusoid) is less than $2.5 \%$. 


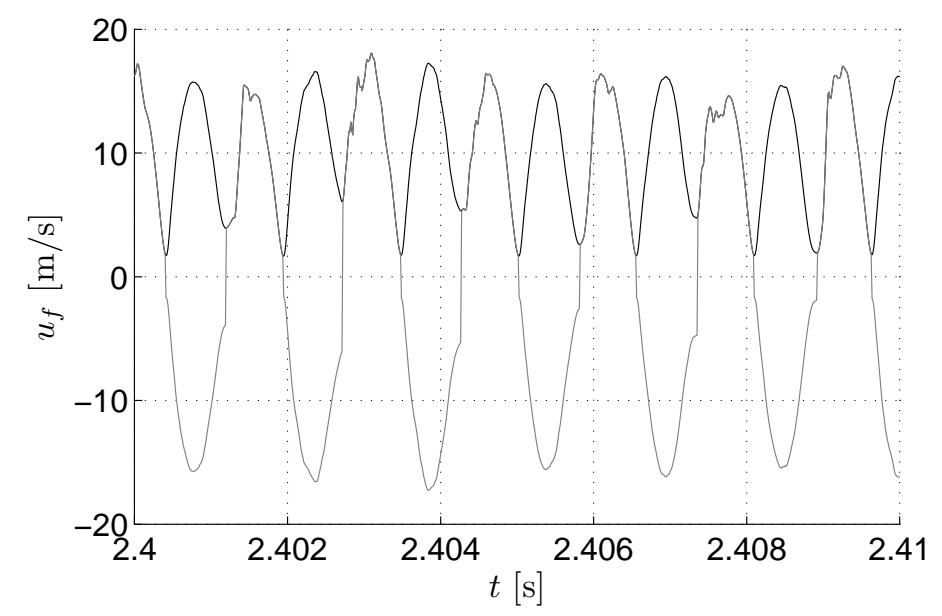

FiguRE 2. Measurement of the jet centre-line velocity: $u_{f},-$, raw; $u_{j}$, - , de-rectified Fourier amplitude.

The forcing amplitude is defined by

$$
C_{\mu}=\frac{u_{j}^{2} A_{j}}{U_{\infty}^{2} A}
$$

where $A_{j}$ is the area of the jet orifice, and $A$ is the area of the base of the model. Considerations of self-similarity imply that the jet momentum flux depends on $\left(u_{j}-U_{\infty}\right)$. However, $U_{\infty}$ is fixed in this experiment and for simplicity therefore, $C_{\mu}$ is defined by (2.1).

The model base is instrumented with 64 static pressure tappings (connected to a 64 channel ESP-DTC pressure scanner) and 11 Endevco $8507 \mathrm{C}$ pressure transducers (only 8 are shown in figure 1) which were sampled at, respectively, $225 \mathrm{~Hz}$ and $40 \mathrm{kHz}$. At each forcing condition, pressure data were acquired for a total of $480 \mathrm{~s}$ over 8 independent experiments, providing a $95 \%$ uncertainty of approximately $6 \%$ and $13 \%$ for the mean and variance of pressure respectively. Pressure measurements are expressed as a pressure coefficient, non-dimensionalised by the free-stream dynamic head.

Random and phase-locked two-component PIV measurements were performed immediately downstream of the jet orifice on the upper and lower separating shear layers simultaneously. A conservative estimate of the PIV spatial resolution, the $-3 \mathrm{~dB}$ cut-off wavenumber of the PIV measurement, gives a cut-off wavelength, $\lambda_{c}=0.7$, where the adjacent vector spacing is $0.2 \mathrm{~mm}$ (Oxlade et al. 2012). Approximately 3500 unforced and 2500 forced (for each forcing condition) independent PIV samples were acquired. Pressure fields were calculated from the randomly sampled PIV measurements by explicit integration of the 2D Reynolds-averaged momentum equations (with the viscous terms omitted) using a single boundary condition. Derivatives are computed using a least-squares finite-difference scheme (see Raffel et al. 2007). Here we ensemble average the solution of $N$ independent, randomly generated integration paths in order to prevent random error in the derivatives smearing the solution in the direction of integration. Furthermore, because the entire domain is calculated from a common origin, only a single boundary condition (located anywhere within the domain) is required. Convergence of the domain-averaged pressure was obtained for $N=1024$ independent integration paths, 


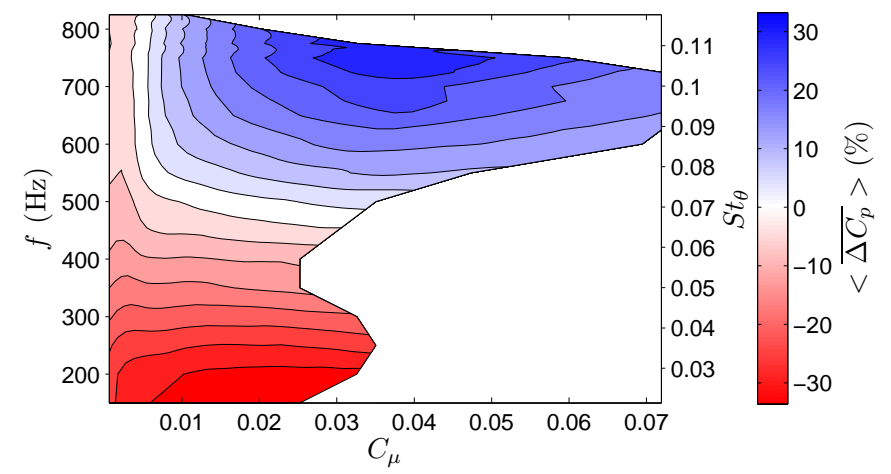

(a)

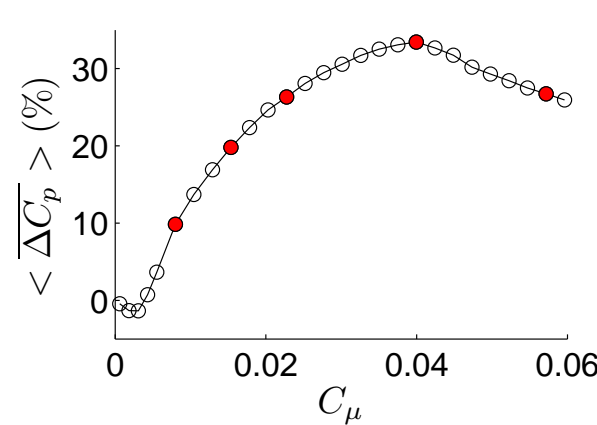

(b)

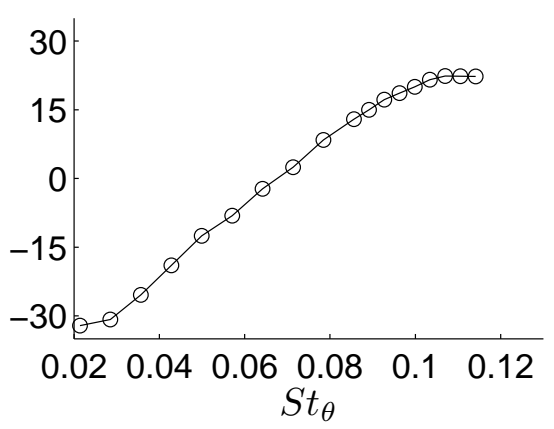

(c)

FiguRE 3. Effect of forcing amplitude and frequency on the mean base pressure coefficient. (a) 2D colour map where contour levels denote $4.2 \%$ intervals. (b) Effect of forcing amplitude at $S t_{\theta}=0.107$. Filled symbols denote values of $C_{\mu}$ used in figure $8(\mathrm{a})$. (c) Effect of forcing frequency at $C_{\mu}=0.018$.

the criterion being $\pm 0.25 \%$ of the free-stream total pressure at inlet to the domain as measured by a Pitot-static tube. Full details on all aspects of the experiment are available in the thesis of Oxlade (2013).

\section{Results and Discussion}

\subsection{Mean base pressure}

The change in mean base-pressure coefficient due to forcing, $\left\langle\overline{\Delta C_{p}}\right\rangle$, calculated as an area-weighted and temporal average, is shown in figure 3(a) as a function of forcing amplitude and frequency. This response map can be loosely divided into pressure decrease and pressure recovery regimes by a horizontal line at $S t_{\theta} \approx 0.07$. Below this frequency, the base pressure reduction occurs due to strong shear-layer amplification with a maximum at $S t_{\theta} \approx 0.021$ (Zaman \& Hussain 1981). For the present experiment $D / \theta=92$, so that turbulence may be regarded as 'small' compared to the large wake structure. This phenomenon is well understood as discussed previously by Qubain (2009); Qubain \& Morrison (2009); Morrison \& Qubain (2009): they also identified the novel pressure recovery regime, appearing here for forcing frequencies, $S t_{\theta} \gtrsim 0.07$, and amplitudes $C_{\mu}>0.005$.

The relationship between blowing coefficient and change in base pressure coefficient at $S t_{\theta}=0.107$ (the frequency at which maximum pressure recovery is observed) is illustrated in figure 3(b). There is a small initial reduction in base pressure at blowing 

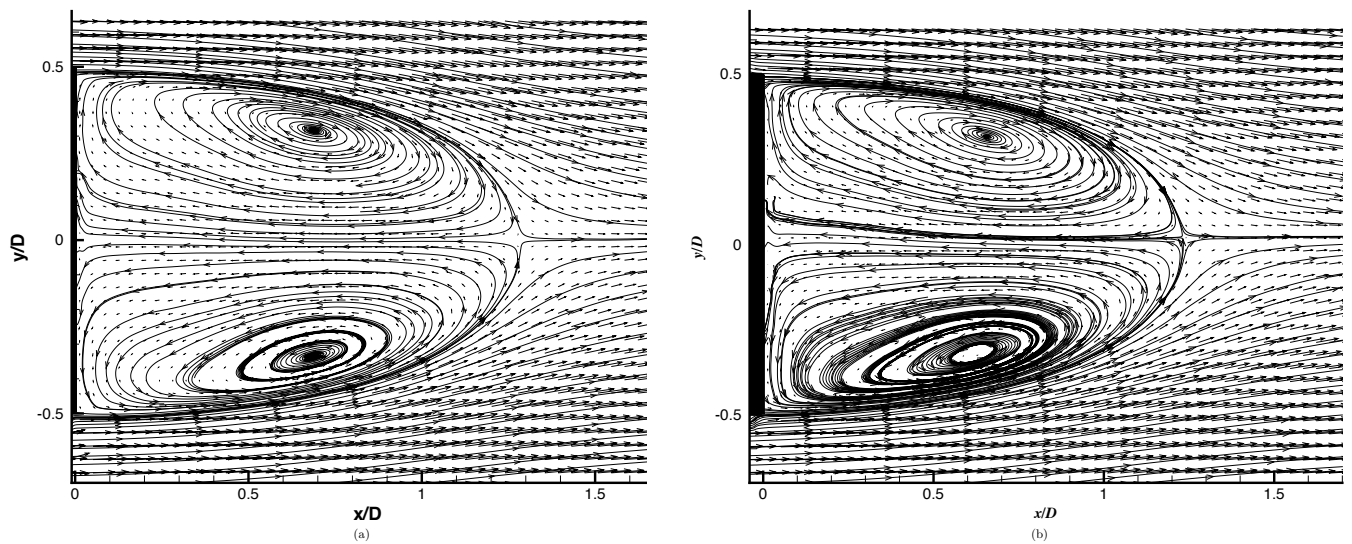

Figure 4. Mean velocity vectors and streamlines, $R e_{\theta}=1700$ at separation: (a) Unforced; (b) Forced $S t_{\theta}=0.076, C_{\mu} \approx 0.03$.

coefficients below $C_{\mu} \approx 0.005$, after which the pressure recovers rapidly. Above $C_{\mu}=0.01$ there is a gradual reduction in gradient, producing a broad region of pressure recovery with a maximum of $33 \%$ at $C_{\mu} \approx 0.04$. For constant amplitude forcing at $C_{\mu}=0.018$, figure $3(\mathrm{c})$ shows that pressure recovery increases linearly up to $S t_{\theta} \approx 0.1$ where it saturates at $\left\langle\overline{\Delta C_{p}}\right\rangle \sim 23 \%$. This suggests that the contours in the upper region of figure 3(a) may extend vertically to provide a wide frequency bandwidth over which pressure recovery can be obtained. This frequency plateau begins at $S t_{\theta} \approx 0.1$, that is, roughly five times the most amplified frequency of the shear layer instability.

\subsection{Mean velocity}

Figure 4 shows mean velocity vectors and streamlines calculated from preliminary PIV vector fields (Qubain 2009) that record the whole recirculation region. Here the vector spacing is $3.6 \mathrm{~mm}$. In the unforced flow, the mean stagnation point occurs at an axial position, $x / D=1.27$, while for the forced case, it is reduced slightly to $x / D=1.23$. This is remarkable in that a drag reduction does not lead to an elongation of the recirculation region, even though the length of the recirculation bubble is shortened when forcing at lower frequencies, $S t_{\theta} \approx 0.025$, and the base pressure decreases. Figure 5 shows the detail close to separation (up to $x / D \approx 0.5$ ) of streamlines and velocity magnitude in the separated shear layer for the unforced and forced case. With the improved resolution (vector spacing $0.2 \mathrm{~mm}$ ), it appears that in the latter case, there is a significant narrowing of the wake and an increase of velocity throughout the shear layer. Both the average length and width of the recirculation bubble are reduced. Interestingly, there is a reversal in the sign of streamline curvature immediately downstream of the trailing edge. This is characteristic of a local rise in pressure at the base, and adjacent to and slightly above the jet exit, there is a small vortex. This is accompanied by a saddle point that forms the corner of the recirculation bubble. These data show that a base pressure rise is associated with a shortening of the bubble and narrowing of the wake due to the local change in the sign of streamline curvature near the trailing edge. 


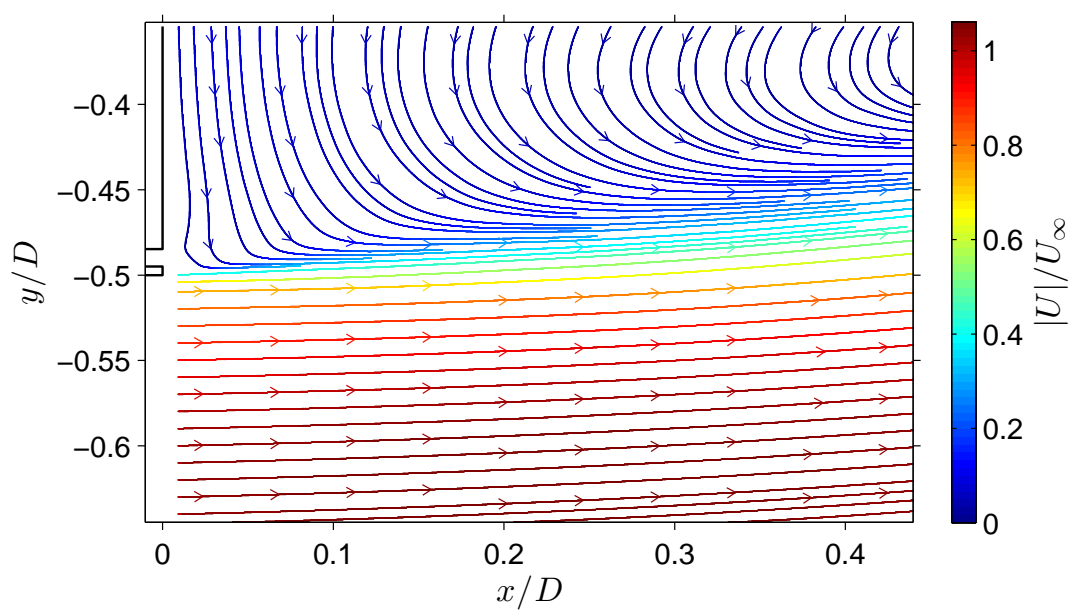

(a)

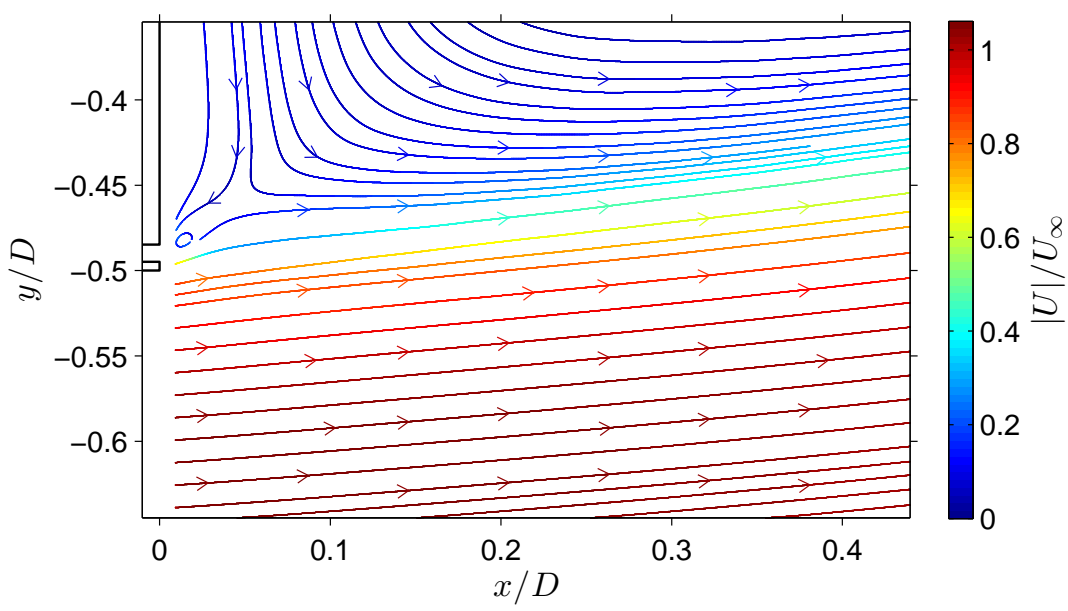

(b)

FigURE 5. Effect of forcing on mean velocity: streamlines coloured by velocity magnitude. (a) Unforced flow; (b) forced flow, $C_{\mu}=0.04, S t_{\theta}=0.107$.

\subsection{Base pressure fluctuations}

Following the method of Rigas et al. (2014b), a Fourier decomposition of the pressure signal in the azimuthal direction gives spatial modes,

$$
p_{m}(r, t)=\frac{1}{(2 \pi)} \int_{0}^{2 \pi} p(r, \phi, t) e^{-i m \phi} \mathrm{d} \phi .
$$




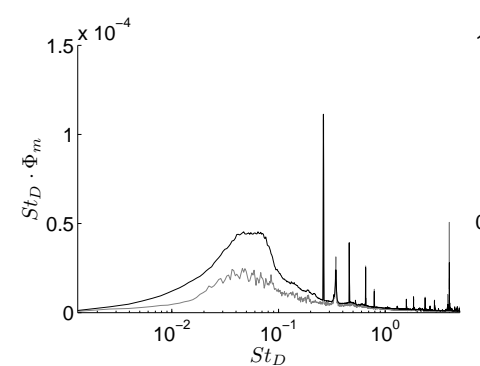

(a)

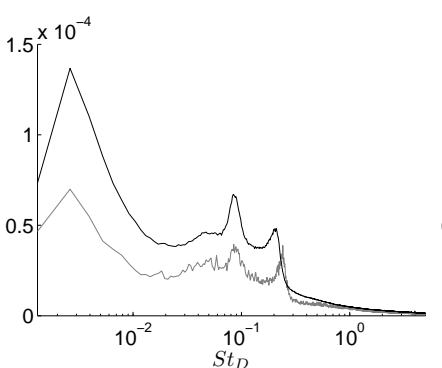

(b)

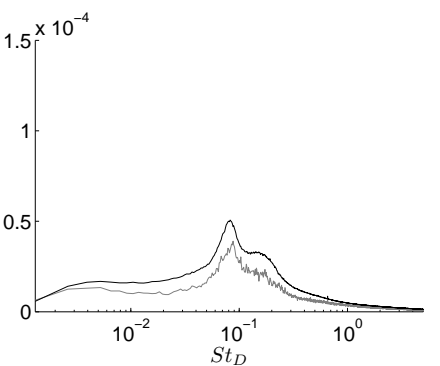

(c)

Figure 6 . Premultiplied pressure spectra by azimuthal mode for the unforced and maximum pressure recovery cases. (a) $m=0$. (b) $|m|=1$. (c) $|m|=2$. — unforced, — forced.

The spectral energy density of each mode $\Phi_{m}(r, S t)$, can be used to identify coherent structures in the wake and when integrated provides the pressure variance per mode,

$$
\overline{p_{m}^{2}}(r)=\int_{0}^{\infty} S t \Phi_{m}(r, S t) \mathrm{d}(\log S t)
$$

Owing to the limited frequency resolution of the static tappings, spectral analysis of the forced flow is performed on the transducer measurements, located at $r=0.3 D$. Figure 5 compares the premultiplied spectral density for the unforced and forced flows, the latter at maximum pressure recovery, $C_{\mu}=0.04$ and $S t_{\theta}=0.107$. The unforced spectral density at this radius is qualitatively similar to the radially averaged result presented in figure 3 of Rigas et al. (2014b).

Within the pressure recovery regime, the effect of high-frequency forcing on pressure fluctuations is a broadband suppression occuring across all modes, without any obvious mode selection. This suggests a strong non-linear coupling between the axisymmetric forcing and all the wake modes. Note that the marked reduction in the $|m|=1$ peak at $S t_{D} \approx 0.2$ confirms that the forcing is able to attenuate the global instability or vortex shedding mode: we also observe a small shift in its frequency which increases with forcing amplitude for $C_{\mu} \gtrsim 0.005$ (see Oxlade 2013).

Figure $7(\mathrm{a})$ shows the mean-square pressure for each mode shape at a forcing frequency of $S t_{\theta}=0.107$ and normalised by the mean-square pressure for the unforced case: it clearly decreases with increasing forcing amplitude. Comparison with figure 4(a) suggests an approximate correlation between the average pressure, $\left\langle\overline{\Delta C_{p}}\right\rangle$, and its variance up to $C_{\mu} \approx 0.04$. However, at larger blowing coefficients, further reduction of meansquare pressure is not matched by an equivalent increase of mean pressure. Further insight is provided by figure $7(\mathrm{~b}, \mathrm{c})$, which shows the pressure variance of the vortexshedding and shear-layer modes respectively. The mean-square pressure associated with the vortex-shedding mode decreases initially for $C_{\mu} \lesssim 0.005$, then remains constant until the maximum pressure recovery at $C_{\mu} \approx 0.04$, after which there is a sharp increase: for larger forcing amplitudes (not shown) this mode is strongly amplified. The mean-square pressure associated with the shear-layer mode (measured by a single outer transducer at $r / D=0.44)$ exhibits a sharp increase just after the optimum forcing amplitude. These data suggest that, above $C_{\mu} \approx 0.04$, the wake modes are amplified by the forcing: in this sense, the natural wake modes compete with the pressure recovery mechanism by increasing entrainment when $C_{\mu} \gtrsim 0.04$. This explains why the pressure fluctuations continue to decrease at larger forcing amplitudes even though the mean pressure recovery is lost. 


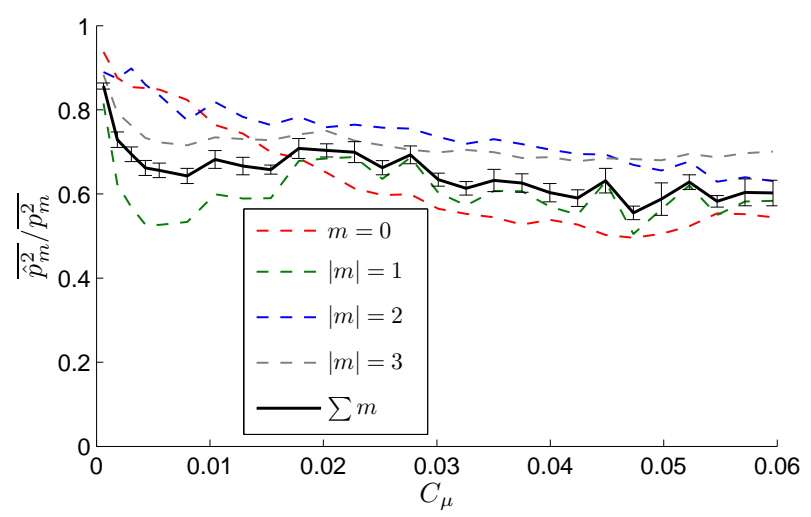

(a)

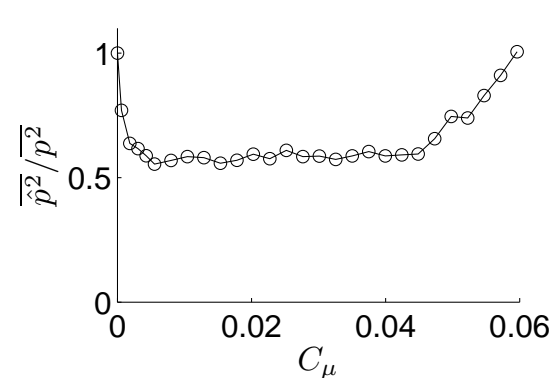

(b)

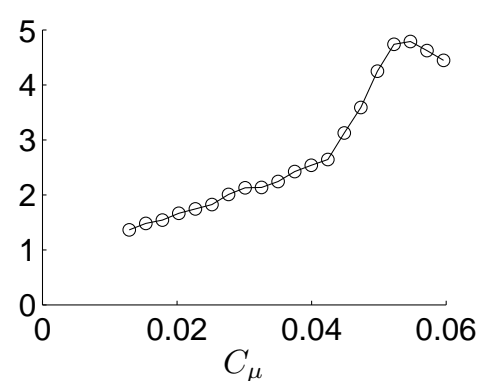

(c)

FIGURE 7. Effect of forcing amplitude on mean-square base pressure for $S t_{\theta}=0.107$. (a) By azimuthal mode shape. (b) Vortex shedding mode. (c) Shear layer mode.

\subsection{Jet structure}

An explanation of this phenomenon is provided in figure 8: the jet produces strong and distinct vortex rings that advect within the shear layer and remain coherent for approximately $x / D<0.2$. Measured over the adjacent vortex positions in figure $8(\mathrm{a})$, their advection velocity is roughly proportional to $C_{\mu}$ and equal to $0.57 U_{\infty}$. They have an initial diameter approximately equal to the jet orifice width. While the vorticity of the primary vortex is about 50 times the mean vorticity of the separating boundary layer, its circulation is only about $10 \%$ of the mean boundary-layer circulation. It is expected that the jet efflux produces a pair of counter-rotating, concentric vortex rings, the outer one with vorticity of opposite sign to that of the boundary layer. Their cancellation leaves the inner vortex ring with vorticity of the same sign as that of the boundary layer.

The magnitude of the pressure recovery is strongly dependent on the strength of the vortex produced by the jet. Figure 8(a) (inset) shows the maximum vorticity of the jet vortex, $\omega_{z} D / U_{\infty}$ as a function of $C_{\mu}$ at $S t_{\theta}=0.107$. The initial increase in forcing amplitude strengthens the primary vortex up to $C_{\mu} \approx 0.04$, where it reaches saturation: inspection of figure 4(b) shows that the maximum pressure recovery coincides with maximum vortex strength. At forcing amplitudes $C_{\mu} \gtrsim 0.04$, the jet vortex is observed to break down immediately into two or more vortical structures (Oxlade 2013). This causes the increased amplification of the wake modes (figure $7 \mathrm{~b}-\mathrm{c}$ ) and consequently, the reduction in pressure recovery.

An important feature of the jet flow topology is highlighted in figure 8(b), which compares the ratio of $Q_{s}$ and $Q_{w}$, respectively, the strain and rotational components of 

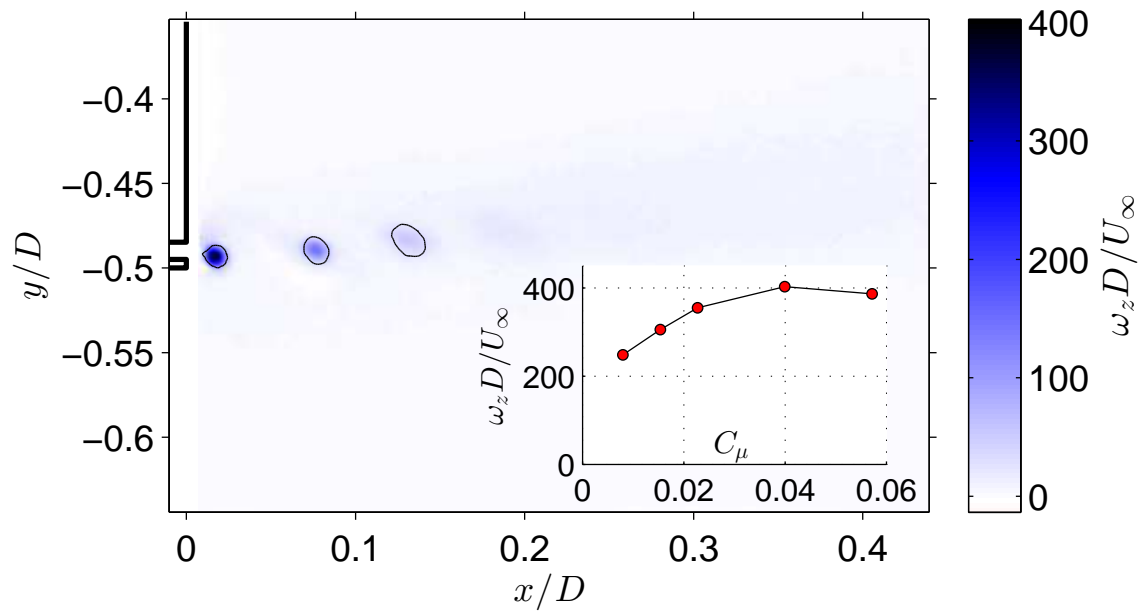

(a)

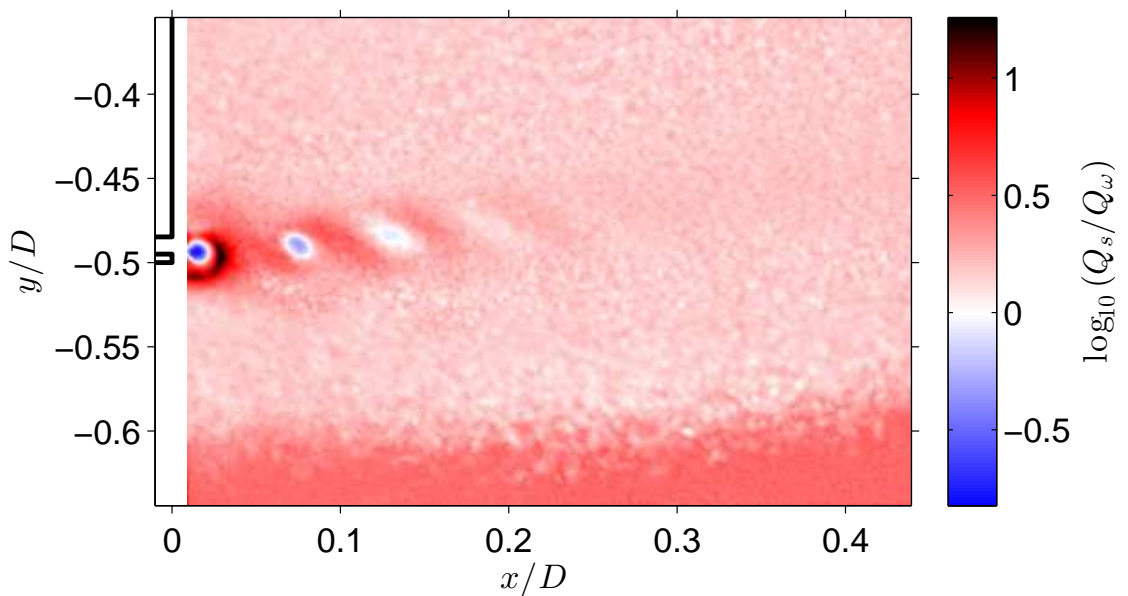

(b)

FiguRE 8. Phase-locked quantities for the maximum pressure recovery $\left(C_{\mu}=0.04, S t_{\theta}=0.107\right)$. (a) Planar vorticity, $\omega_{z} D / U_{\infty}$, with vortices identified by single contour of swirl strength, $\lambda_{i}=10$. Inset shows peak vorticity, $\omega_{z}$ of the initial vortex as a function of $C_{\mu}$ corresponding to conditions in figure 3(b) denoted by filled symbols. (b) Corresponding $Q_{s} / Q_{\omega}$.

the second invariant, $Q$, of the strain rate tensor, $s_{i j}$ :

$$
Q=Q_{s}+Q_{\omega}=-\frac{1}{2} s_{i j} s_{j i}+\frac{1}{4} \omega_{i} \omega_{i}
$$


where in the $(x, y)$-plane,

$$
s_{x y}^{2}=\left(\frac{\partial u}{\partial x}\right)^{2}+\frac{1}{2}\left(\frac{\partial u}{\partial y}+\frac{\partial v}{\partial x}\right)^{2}+\left(\frac{\partial v}{\partial y}\right)^{2}
$$

and

$$
\frac{1}{2} \omega_{z}^{2}=\frac{1}{2}\left(\frac{\partial u}{\partial y}-\frac{\partial v}{\partial x}\right)^{2}
$$

In general, the left-hand side of (3.3) is the small difference between two large quantities because shear layer turbulence consists predominantly of vortex sheets or internal shear layers which have nearly opposite $s_{i j}$ and $\omega_{i}$ and, being approximately plane with nearly straight streamlines, do not contribute significantly to the pressure. This is not the case in figure 8(b): where $Q_{s} / Q_{\omega}>>1, Q \approx Q_{s}$ and the local flow is predominantly a straining motion with strong dissipation. Alternatively, where $Q_{s} / Q_{\omega}<<1, Q \approx Q_{\omega}$ and the local flow is dominated by strongly rotating but weakly dissipative motion. Figure 8 (b) shows that the distinction between these two topologies is unambiguous since the jet structure comprises strongly rotational vortex cores immediately surrounded by, and joined by regions of nearly pure shear. Consequently, in regions where the flow is dominated by these structures, the sign of $Q$ is sufficient to distinguish between rotation and shear.

\subsection{The pressure recovery mechanism}

The static pressure field for the unforced and forced flows is compared in figure 9: in the forced case the jet creates a thin region of very low pressure, the minimum of which is coincident with the trajectory of the jet vortex cores. The resulting pressure recovery is clearly visible throughout the flow field, encompassing both the base region and the outer side of the shear layer for $x / D>0.3$. Figure $9(\mathrm{~b})$ shows that the computed pressure distribution is in close agreement with the transducer measurements on the base of the body.

Bradshaw \& Koh (1981) have shown that the Poisson equation for pressure fluctuations may be written

$$
-\frac{\nabla^{2} p}{\rho}=-2 Q=s_{i j} s_{j i}-\frac{1}{2} \omega_{i} \omega_{i},
$$

where the right-hand side is decomposed into the sum of symmetric (straining) and antisymmetric (rotational) components of the strain rate tensor. Although this decomposition is not unique (Adrian 1982), the former may be taken to represent the non-local generation of pressure by stagnation points in the instantaneous streamline pattern ("eddy collisions") while the rotational term (enstrophy) indicates local regions of low pressure created by centripetal acceleration.

Figure 10 compares the distribution of time-averaged $-2 Q((3.6)$ in the $(x, y)$-plane) for the unforced and forced flow at maximum pressure recovery. The vertical profiles (figure $10 \mathrm{~b}$ ) show $-2\langle Q\rangle$, the second invariant averaged over a short streamwise region near the jet. This clearly shows that the forced wake is dominated by a narrow region of large enstrophy $(2\langle Q\rangle \gg 0)$ bounded on each side by a strong shear layer $(2\langle Q\rangle \ll 0)$, associated with high dissipation and responsible for a reduction in entrainment. Specifically, the term $\frac{\overline{\partial u}}{\partial y} \frac{\partial v}{\partial x}$ determines the sign of $Q$ and therefore whether a region is one of large, nearly irrotational dissipation or one of large enstrophy. 


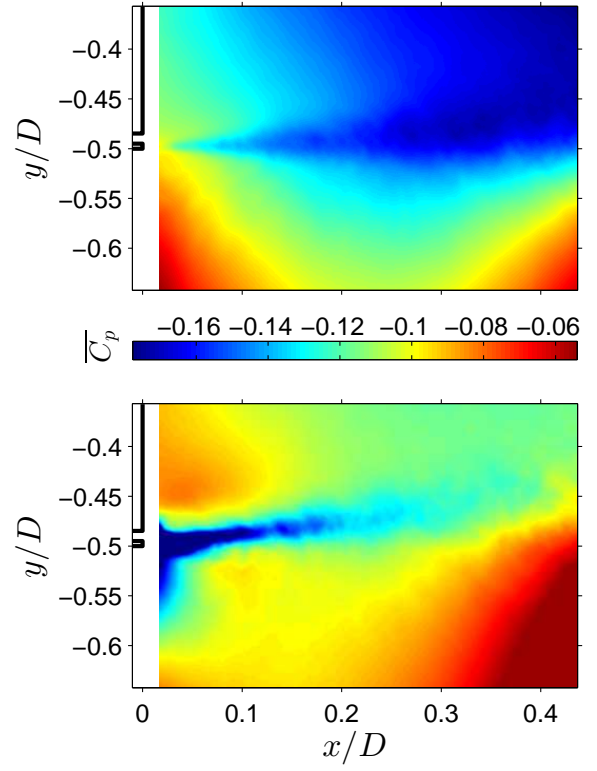

(a)

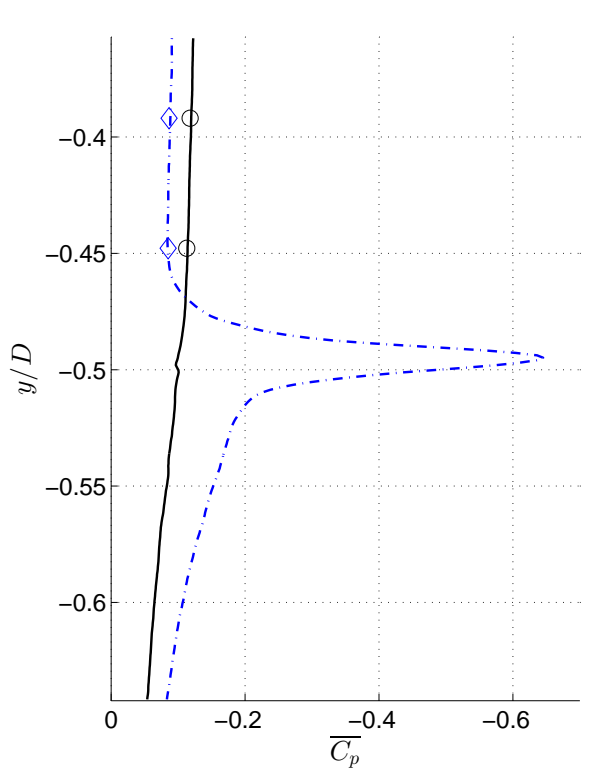

(b)

Figure 9. Time-averaged pressure coefficient, $\overline{C_{p}}$, obtained as integral of velocity field in $(x, y)$-plane: colour scale limits for the forced flow are truncated to match the limits of the unforced flow. (a) pressure maps unforced (top) and forced (bottom), $S t_{\theta}=0.107, C_{\mu}=0.04$ : note minimum $\overline{C_{p}} \approx-0.6$. (b) Corresponding line graphs for $x / D=0.0$ : $\longrightarrow$, unforced; $-\cdot-\cdot-$, forced. Symbols denote corresponding base pressure transducer measurements.

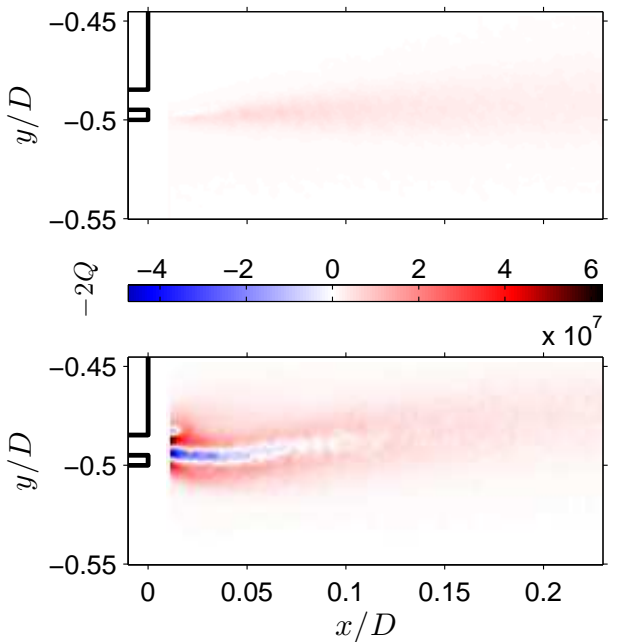

(a)

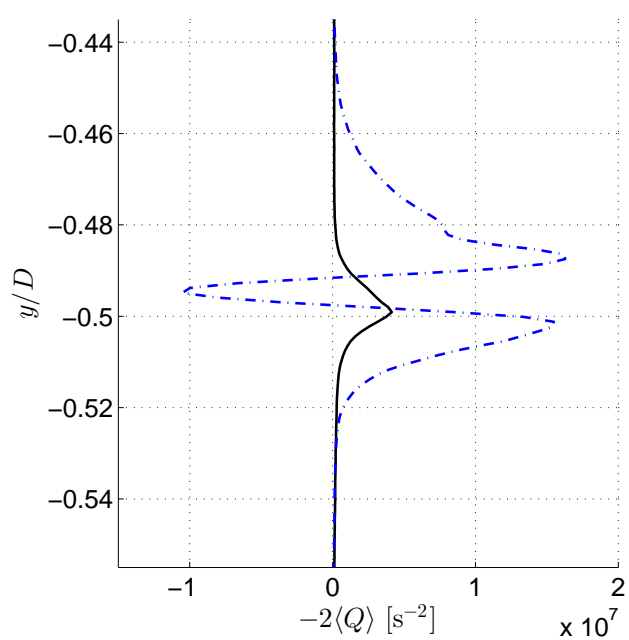

(b)

Figure 10. Comparison of the second invariant, $-2 Q$, in the forced and unforced flows. (a) Time averaged colour maps, $C_{\mu}=0.04$ (top) and unforced (bottom). (b) Vertical profiles of $-\langle 2 Q\rangle$ averaged for $0.01<x / D<0.07$. - , unforced; $-\cdot \cdot \cdot-$, forced. 


\section{Discussion and Conclusions}

The effect of pressure recovery behind a bluff body with a sharp trailing edge is generic in that we have also been able to demonstrate it for turbulent separation from a bluff body of rectangular cross section (Cabitza 2013) as well as that from a backward-facing step (Qubain 2007). The unifying feature of these experiments is that the entrainment and therefore the growth rates of these wakes have been reduced, with a concomitant reduction in pressure drag. The mechanism by which pressure is recovered in the wake appears to be the creation of a narrow region of strong enstrophy bounded on each side by a very strong shear layer: the region of large enstrophy is coincident with the vortex core trajectory, and since each vortex is instantaneously demarcated by a region of very strong shear, its advection results in a time-averaged shear layer on each side. These are associated with high dissipation and responsible for the reduction in entrainment and hence drag on the body.

These effects appear to induce large scale changes in the wake, where the length of the recirculation region is slightly reduced by high-frequency forcing. Near the base of the body, there is a point of inflection in the separating streamline which leads to a narrowing of the wake. It seems likely that the models proposed by Roshko (1993) and Gerrard (1966) would require modification in order to describe this effect. Further studies are underway involving the simultaneous measurement of base pressure and the vorticity field.

We are indebted to Ferrari S.p.A. and to EPSRC (Grant number EP/I005684) for financial support.

\section{REFERENCES}

AChenbach, E. 1974 Vortex shedding from spheres. J. Fluid Mech. 62, 209-221.

Adrian, R. J. 1982 Comment on "a note on Poisson's equation for pressure in a turbulent flow". Phys. Fluids 25, 577.

Barros, D., Ruiz, T., Borée, J. \& NoAck, B.R. 2014 Control of a three-dimensional blunt body wake using low and high frequency pulsed jets. Int. J. Flow Control .

Berger, E., Scholz, D. \& Schumm, M. 1990 Coherent vortex structures in the wake of a sphere and a circular disk at rest and under forced vibrations. J. Fluids 86 Struct. 4, 231-257.

Bradshaw, P. \& Koh, Y. M. 1981 A note on Poisson's equation for pressure in a turbulent flow. Phys. Fluids 24, 777.

Cabitza, S. 2013 Active Control of the Wake from a Rectangular-Sectioned Body. PhD dissertation, Imperial College, Department of Aeronautics.

Choi, H., Jeon, W-P. \& Kim, J. 2008 Control of flow over a bluff body. Annu. Rev. Fluid Mech. 40, 113-139.

Corrsin, S. \& Kistler, A. L. 1955 Free-stream boundaries of turbulent flows. Technical Report, NACA TN-1244.

Dahan, J. A., Morgans, A. S. \& Lardeau, S. 2012 Feedback control for form-drag reduction on a bluff body with a blunt trailing edge. J. Fluid Mech. 704, 360-387.

Fuchs, H. V., Mercker, E. \& Michel, U. 1979 Large-scale coherent structures in the wake of axisymmetric bodies. J. Fluid Mech. 93, 185-207.

Gaster, M., Kit, E. \& Wygnanski, I. 1985 Large-scale structures in a forced turbulent mixing layer. J. Fluid Mech. 150, 23-39.

Gerrard, J. H. 1966 The mechanics of the formation region of vortices behind bluff bodies. $J$. Fluid Mech. 25 (2), 401-413.

Glezer, A. \& Amitay, M. 2002 Synthetic jets. Annu. Rev. Fluid Mech. 34, 503-529.

Grandemange, M., Gohlke, M. \& Cadot, O. 2013 Turbulent wake past a three-dimensional blunt body. Part 1. Global modes and bi-stability. J. Fluid Mech. 722, 51-84. 
Heenan, A. F. \& Morrison, J. F. 1996 Passive control of backstep flow. Exptl. Thermal Fluid Sci. 13, 211-222.

Heenan, A. F. \& Morrison, J. F. 1998 Passive control of pressure fluctuations generated by separated flow. AIAA J. 36, 1014-1022.

Ho, C.-M. \& Huerre, P. 1984 Perturbed free shear layers. Annu. Rev. Fluid Mech. 463, $365-424$.

Huerre, P. 2000 Open shear flow instabilities. In Perspectives in Fluid Dynamics (ed. G. K. Batchelor, H. K. Moffatt \& M. G. Worster). Cambridge: Cambridge University Press.

KANG, S. \& ChOI, H. 2002 Suboptimal feedback control of turbulent flow over a backward facing step. J. Fluid Mech. 463, 201-227.

Kim, J. \& ChOI, H. 2005 Distributed forcing of flow over a circular cylinder. Phys. Fluids 17, 033103.

Monkewitz, P. A. 1988 A note on vortex shedding from axisymmetric bluff bodies. J. Fluid Mech. 192, 561-575.

Morrison, J. F. \& Qubain, A. 2009 Control of an axisymmetric turbulent wake by a pulsed jet. In Advances in Turbulence XII - Proceedings of the12th EUROMECH European Turbulence Conference (ed. B. Eckhardt), Springer Proceedings in Physics, vol. 132, pp. 225-228. Springer-Verlag.

Oxlade, A. R. 2013 High-frequency Periodic Jet Forcing of a Turbulent Axisymmetric Wake. $\mathrm{PhD}$ dissertation, Imperial College, Department of Aeronautics.

Oxlade, A. R., Valente, P. C., Ganapathisubramani, B. \& Morrison, J. F. 2012 Denoising of time-resolved PIV for accurate measurement of turbulence spectra and reduced error in derivatives. Exps. Fluids 53, 1561-1575.

Parezanovic, V. \& CAdot, O. 2012 Experimental sensitivity analysis of the global properties of a two-dimensional turbulent wake. J. Fluid Mech. 693, 115-149.

Philip, J. \& Marusic, I. 2012 Large-scale eddies and their role in entrainment in turbulent jets and wakes. Phys. Fluids 24, 055108.

Qubain, A. 2007 Separated Shear Flow Manipulation Using Periodic Forcing. Transfer report, Imperial College, Department of Aeronautics.

Qubain, A. 2009 Active Control of a Turbulent Bluff Body Wake. PhD dissertation, Imperial College, Department of Aeronautics.

Qubain, A. \& Morrison, J. F. 2009 Active control of an axisymmetric turbulent wake. In KATnet II Conference on Key Aerodynamic Technologies. Bremen, May 12-14.

Raffel, M., Willert, C., Wereley, S. \& Kompenhans, J. 2007 Particle Image Velocimetry: A Practical Guide, 2nd edn. Springer.

Rigas, G., Morgans, A. S., Brackston, R. D. \& Morrison, J. F. $2014 a$ Diffusive dynamics of turbulent axisymmetric wake flows. In preparation .

Rigas, G., Oxlade, A. R., Morgans, A. S. \& Morrison, J. F. $2014 b$ Low-frequency dynamics of a turbulent axisymmetric wake. J. Fluid Mech. 755, R5.

Roshko, A. 1993 Perspectives on bluff body aerodynamics. J. Wind Eng. Ind. Aero. 49, 79-100.

Strykowski, P. J. \& Sreenivasan, K. R 1990 On the formation and supression of vortex 'shedding' at low reynolds numbers. J. Fluid Mech. 218, 71-107.

TANEDA, S. 1978 Visual observations of the flow past a sphere at Reynolds numbers between $10^{4}$ and $10^{6}$. J. Fluid Mech. 85, 187-192.

Townsend, A. A. 1976 The Structure of Turbulent Shear Flow, 2nd edn. Cambridge University Press.

TuRner, J. S. 1986 Turbulent entrainment: the development of the entrainment assumption, and its application to geophysical flows. J. Fluid Mech. 173, 431-471.

Vukasinovic, B., Rusak, Z. \& Glezer, A. 2010 Dissipative small-scale actuation of a turbulent shear layer. J. Fluid Mech. 656, 51-81.

Westerweel, J., Kukushima, C., Pedersen, J. M. \& Hunt, J. C. R. 2005 Mechanics of the turbulent-nonturbulent interface of a jet. Phys. Rev. Lett. 95, 174501.

Zaman, K. B. M. Q. \& Hussain, A. K. M. F. 1981 Turbulence suppression in free shear flows by controlled excitation. J. Fluid Mech. 103, 133-159. 